\title{
La qualité de la traduction spécialisée : révision dans les contextes pédagogique et professionnel
}

\author{
Dima EL HUSSEINI \\ Professeur-adjoint \\ Faculté des Langues Appliquées \\ Université Française d'Egypte
}

\section{Résumé}

La présente étude se propose d'analyser l'évaluation de la qualité de la traduction spécialisée dans le contexte pédagogique. Il s'agit, dans un premier temps, d'étudier les normes de management de la qualité dans le contexte professionnel à travers l'analyse de l'évaluation de la qualité de la traduction des étudiants de la faculté des Langues Appliquées de l'Université Française d'Egypte dans le cadre des rapports de stage de traduction. Dans un deuxième temps, il s'agit d'analyser la démarche effectuée dans les cours de traduction spécialisée dans les combinaisons linguistiques arabe $\leftrightarrow$ français. Dans cette étude, il s'agit de la révision de la traduction dans le milieu universitaire en tenant compte des modalités d'évaluation professionnelle pratiquée dans les institutions ayant accueilli les étudiants pour une période de six semaines de stage. Cette analyse permet d'établir, dans une perspective formative, une comparaison entre les critères de qualité dans le milieu professionnel et ceux dans le milieu académique, en étudiant la notion d'acceptabilité dans son rapport avec la notion de l'erreur. La réflexion que nous menons dans cette étude s'interroge sur les moyens qui permettent l'évolution des critères d'évaluation de la qualité de la traduction dans le cadre de l'enseignement suite à l'analyse de l'expérience professionnelle des étudiants. Notre analyse porte, dans une optique traductologique, sur des exemples de textes spécialisés travaillés dans les cours de traduction, d'une part, et dans le cadre des stages, d'autre part. L'observation des résultats obtenus montre les répercussions pédagogiques de l'étude de l'évaluation de la qualité de la traduction spécialisée dans le monde professionnel.

Mots-clés : qualité en traduction, traduction spécialisée, traduction professionnelle, traductologie, révision.

$$
\text { ملذص البحث باللغة العربية }
$$

يهدف هذا البحث إلى تحليل تقويم جودة الترجمة التخصصية في المجال التربوي. ويتناول دراسة معايير

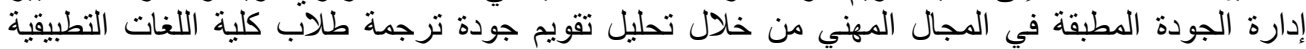

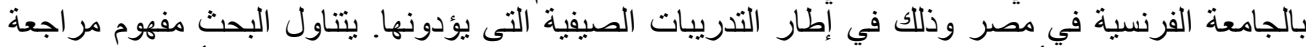

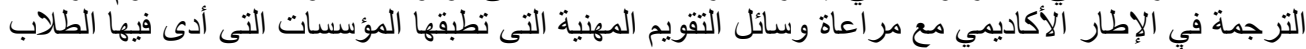

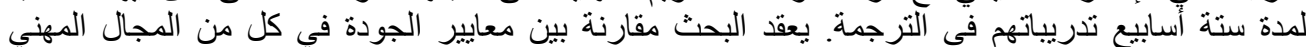

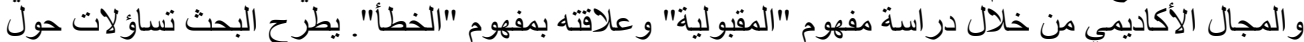

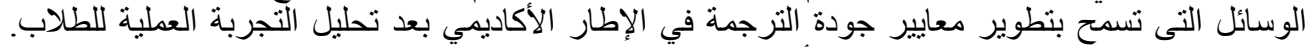

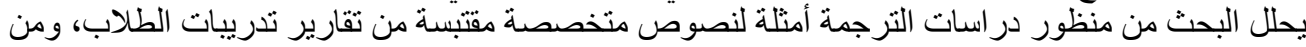
النصوص التى تدرس في محاضر ات الترجمة (فرنسي شعربي). 
La qualité de la traduction spécialisée : révision dans les contextes pédagogique et professionnel

\section{Introduction}

L'objectif de cette étude est de montrer que la formation professionnalisante permet aux étudiants de devenir opérationnels sur le marché du travail. Le stage se présente comme la première expérience professionnelle des étudiants sur le marché. Cette étude, se focalise, dans une perspective traductologique sur la révision de la traduction des étudiants tant en milieu pédagogique que professionnel à travers des exemples tirés, d'une part, des cours de traduction spécialisée de la faculté des Langues Appliquées de l'Université française d'Egypte et des rapports de stages des étudiants, d'autre part.

La réflexion que nous menons dans cette étude s'interroge sur la qualité de la traduction fournie par nos étudiants: répond-t-elle aux critères de qualité des institutions qui les accueillent en stage? Comment l'analyse de la première expérience professionnelle des étudiants et le contact direct des enseignants avec les tuteurs contribuent-ils à définir les moyens qui permettent l'évolution des critères de la révision de la traduction dans le cadre de l'enseignement?

D'abord, il sera question de la notion de qualité en traduction. Ensuite, les notions de révision, d'erreur et d'acceptabilité seront analysées dans les contextes pédagogique et professionnel. Enfin, les résultats de l'observation montrent l'orientation des enseignants et des tuteurs de stage en matière de révision assurant la qualité de la traduction.

\section{Méthodologie de travail}

La présente étude repose sur deux analyses. La première est celle des rapports de stages de $3^{\mathrm{e}}$ année. La deuxième est celle des devoirs de traduction des étudiants de $3^{\mathrm{e}}$ année et de $4^{\mathrm{e}}$ années. Vingt-sept rapports de stage ont été analysés sur une période de 11 ans (de 2005 à 2016). Cette démarche s'inscrit dans le cadre du projet de promotion du département de traduction spécialisée entamé en 2006 au sein de la Faculté des Langues Appliquées. Cette analyse se focalise sur les acquis de l'expérience professionnelle des étudiants à travers deux axes. D'une part, l'étude de l'influence de la formation professionnalisante sur cette expérience. D'autre part, les répercussions de cette expérience sur la mise à jour du contenu pédagogique pour former des traducteurs qui répondent aux critères de qualité prévalant sur le marché du travail aux niveaux tant local qu'international.

Les vingt-sept rapports sélectionnés pour notre étude représentent un intérêt particulier. Ils ont été effectués dans des lieux qui représentent les métiers de la traduction: traducteur, terminologue, localisateur, rédacteur web, traducteurjournaliste, traducteur de veille. Ils portent sur les trois combinaisons linguistiques enseignées au sein des Langues Appliquées: arabe $\leftrightarrow$ français, arabe $\leftrightarrow$ anglais et anglais $\rightarrow$ français (1). Ce corpus couvre des secteurs d'activités variés dont les affaires, le commerce, le marketing, les médias, la médecine et la technologie. Il 
s'agit d'institutions publiques et privées, d'organisations internationales, d'organisations non-gouvernementales, des bureaux de traduction, des agences de localisation, des cabinets d'avocat internationaux et des médias.

L'analyse des rapports, effectuée chaque année, est souvent suivie d'une synthèse regroupant les constatations et les propositions pour améliorer la qualité de l'enseignement dispensé en traduction spécialisée. Ce dernier doit être en phase avec les attentes du marché. Le contact direct avec les tuteurs de stage est une étape clé dans cette démarche. Ce contact a lieu soit par téléphone soit en invitant les tuteurs à faire un témoignage lors de la célébration de la Journée du traducteur (2) au sein de la Faculté.

L'analyse des rapports de stage tient compte du type de l'institution d'accueil, de son emplacement (niveau local, régional ou international), de son domaine d'activité, des combinaisons linguistiques travaillées par l'étudiant, du nombre de mots traduits, des supports et des outils.

L'analyse des rapports de stage (dans la combinaison linguistique arabe $\leftrightarrow$ français, arabe $\leftrightarrow$ anglais et anglais $\leftrightarrow$ français) et des devoirs maison de traduction (dans la combinaison linguistique français $\leftrightarrow$ arabe) porte sur les types de textes traduits, les exercices de traduction effectués, les types d'erreurs, leurs sources et la révision du tuteur.

\section{Qualité en traduction :}

La qualité est le premier souci du client. Examinons, d'abord, la définition de la qualité dans la norme expérimentale concernant le projet de traduction ISO 11669 de 2012 : « la qualité peut être définie comme étant le degré de conformité du produit de la traduction aux spécifications de projet»(3). Ces derniers "définissent et orientent [...] l'ensemble du projet de traduction à évaluer. » (4).

La notion de qualité a évolué. Au début, elle concernait le contrôle de la traduction (le produit fini) par la révision; ensuite, elle a inclus tout «le processus de conception et de développement qui aboutit à la réalisation d'un produit» (5). Dans son aperçu sur l'évolution des critères de la qualité, Guidère (6) introduit les trois critères d'évaluation cités par Nida (1964) : «l'efficacité générale du processus de communication ; [...] la compréhension de l'intention qui sous-tend le message ; [et] la production d'une réponse analogue chez les deux publics (source et cible) » (7). L'approche textuelle de la qualité de Reiss (1971) évolue vers l'approche fonctionnelle de la théorie du skopos (8). Dans cette dernière, les critères d'évaluation des traductions ne dépendent pas du type de texte mais du but (skopos) du texte à traduire (Reiss et Vermeer, 1984). Comment ce but est-il défini? Certes, par la définition du «destinataire [...] caractérisé par des connaissances et des attentes modelées par sa propre culture. » (9). L'évaluation de la traduction se fait en fonction de son adaptation au but prédéfini (10). Ce but est mentionné dans le cahier 
La qualité de la traduction spécialisée : révision dans les contextes pédagogique et professionnel

des charges, « déterminé par la négociation entre le traducteur et le commanditaire. » (11).

Dans la réalité pratique, les critères de qualité sont relatifs. Nombre d'éléments y sont pris en compte dont «le temps, le lieu, les circonstances de la communication, le genre de traduction, la nature du texte et sa finalité et, enfin, le destinataire. » (12). Les deux éléments primordiaux liés aux critères de la qualité sont l'exactitude et la lisibilité (13). Il s'agit de s'assurer que le sens est «exact». Autrement dit, transmis sans suppression ni ajout. La lisibilité signifie que le texte est facile à comprendre (14).

Un texte traduit est jugé « de qualité » lorsqu'il satisfait le client. Mais, comment s'assurer que la qualité fournie « [correspond] à l'attente du client en matière de qualité. »? (15). En effet, cette attente est, partiellement, définie dans le cahier des charges dans lequel le client peut préciser un choix particulier concernant une terminologie ou une phraséologie qu'il exige. C'est la prise en compte de « "facteurs environnementaux" comme le clientélisme (soit le service sur mesure axé sur le client, notion clé des années 90) qui conditionnent le produit fini et son évaluation. » (16). C'est dans ce contexte que la révision doit tenir compte du cahier des charges.

La notion de qualité est liée aux normes. Les normes désignent les conventions de la langue, les exigences de terminologie et de style ; elles renvoient aussi aux «normes techniques ou industrielles (« standards »), émises par les organismes de normalisation comme l'ISO [...] qui encadrent non seulement la production industrielle mais aussi les prestations de service en traduction et les pratiques en terminologie » (17). A cela s'ajoutent les normes socio-culturelles étudiées par Toury (1995).

La notion de qualité intéresse à la fois le monde académique et le monde professionnel. Ils partagent le même objectif : «la sensibilisation plus ou moins poussée de divers publics à la qualité de la traduction comme processus et comme produit. L'une et l'autre s'entendent sur le fait que la formation professionnelle est garante elle aussi de la qualité de la prestation »(18).

\subsection{Méthodologie de la traduction : gage de qualité ?}

Les cours de traduction de $3^{\mathrm{e}}$ année (Licence pour notre partenaire Sorbonne Nouvelle-Paris 3) et ceux de traduction de textes économiques juridiques et techniques de $4^{\mathrm{e}}$ année (Master 1 pour notre partenaire Sorbonne Nouvelle-Paris 3) s'articulent autour de la méthodologie de la traduction et celle de la recherche documentaire appliquée à la traduction. Il s'agit d'une approche méthodologique axée sur la pratique de la traduction dans ses différents stades. Autrement dit, sur le processus de traduction lui-même. Comment l'enseignant juge-t-il de la qualité de la traduction des étudiants? Dans ce contexte, il s'agit aussi bien de la qualité des 
textes traduits dans le cadre des cours de traduction spécialisée que de la qualité du contenu textuel traduit dans le cadre des projets pédagogiques multimédias (19).

En ce qui concerne le déroulement du cours de traduction, la phase de pré-traduction est évaluée à travers la lecture active, la recherche documentaire (ciblée), la lecture de textes parallèles, la recherche terminologique et phraséologique, la déverbalisation et la réexpression. L'étape de déverbalisation est extrêment importante. Elle consiste à extraire le sens brut, dénué de tout aspect linguistique (20). La démarche de pré-traduction et le texte traduit sont évalués en fonction de la consigne donnée par l'enseignant avant d'entamer le processus de traduction. La consigne est souvent inspirée des rapports de stage qui reflètent la diversité des situations professionnelles auxquelles les stagiaires se sont confrontés.

Dans les cours, les exercices de traduction sont variés et ne se limitent pas à la traduction. Les étudiants s'entrainent à la synthèse croisée (traduction sous forme de synthèse de documents dans la langue cible), vulgarisation dont le processus se focalise sur les procédés mis en œuvre pour traduire un contenu spécialisé adressé à un destinataire non spécialiste. Ils pratiquent la traduction de veille qui consiste à rechercher et à analyser l'information multilingue concernant un sujet bien précis dans plusieurs langues et à rédiger un compte rendu de recherches dans une langue définie (21). A travers ces exercices, le choix de la forme et du registre de langue du texte à traduire doivent correspondre à la consigne donnée par l'enseignant (22). Les textes sources choisis en $3^{\mathrm{e}}$ année sont des textes d'analyse et semi-spécialisés. Ceux de $4^{\mathrm{e}}$ année sont des textes spécialisés comme les rapports, les bilans et les conventions.

Le suivi de l'application de la méthodologie de la traduction permet d'évaluer le processus de traduction et d'assurer le progrès de l'étudiant : « le traitement réservé de l'erreur de l'apprenant est fondamental : si la méthode d'enseignement appliquée ne lui permet pas d'apprendre à éviter de la réitérer en favorisant chez lui l'acquisition d'une méthodologie, l'étudiant ne pourra faire aucun progrès » (23). Avançons dans ce sens l'exemple d'une étudiante qui a effectué son stage à l'UNESCO en 2015. La stagiaire a assuré la révision linguistique de la brochure du musée nubien en Haute Egypte, traduite de l'anglais vers l'arabe sur laquelle se trouve une carte géographique de l'Egypte. Sensibilisée vis-à-vis de la recherche documentaire ciblée en vue d'une traduction, la stagiaire a vérifié aussi la carte et a découvert l'absence des deux villes «Halayeb et Chalatine », objet de litige avec le Soudan. Oubli qui risque de susciter une crise diplomatique. D'où l'importance d'une vigilance constante.

La démarche du cours de traduction (et celle des projets pédagogiques) se focalise sur les tâches d'assurance qualité en cours de processus décrites dans la norme expérimentale ISO, 11669 de 2012. Il s'agit de cinq étapes : l'autovérification, la révision, la relecture d'expert, la mise en forme et la correction d'épreuves (24). 
La qualité de la traduction spécialisée : révision dans les contextes pédagogique et professionnel

Dans un premier temps, chaque étudiant assure la révision de son propre travail de traduction à travers la comparaison de la traduction à l'original. Il s'assure qu'il a rendu le sens sans suppression ni ajout par rapport à l'original. Dans un deuxième temps, la révision est pratiquée par les étudiants en alternance. Chaque étudiant révise le travail de son camarade en trois étapes : en comparant le texte traduit au texte source, en lisant le texte traduit uniquement «pour assurer le contrôle terminologique (vérification de la cohérence terminologique du texte cible à l'aide souvent d'une base de données terminologiques que l'équipe de terminologie a créé) et enfin, en relisant le texte traduit « de point de vue morphologique et syntaxique » (25). La relecture d'expert se limite, dans le cadre des cours, à la consultation d'un spécialiste pour valider la terminologie. Certes, il n'est pas évident d'avoir pour chaque texte traduit une lecture de la part d'un expert quoiqu'elle soit primordiale pour «(évaluer) le texte cible en accordant une attention particulière aux conventions liées au domaine et au type de texte»(26). Mais les étudiants sont sensibilisés vis-à-vis de l'importance de cette étape dans la démarche qualité en matière de traduction et la consultation d'un spécialiste pour la validation des termes est exigée. Le chef d'équipe désigné se charge de la mise en forme du texte traduit avant la correction d'épreuve qui concerne la qualité de la typographie comme les coquilles et les fautes relatives à l'impression (27).

L'exercice de révision dans la formation des traducteurs les aide à « améliorer et [à] contrôler la qualité des textes » (28). A travers cette démarche, l'accent est mis sur la qualité au niveau du processus de traduction engagé dans le cours de traduction et dans le cadre des projets pédagogiques. L'évaluation faite par l'enseignant est liée aux objectifs d'apprentissage: «aucun processus d'évaluation n'a de sens indépendamment des objectifs d'apprentissage. » (29).

\subsection{Notion de révision}

La révision consiste à porter « un jugement sur la qualité d'un texte » (30). Dans le milieu professionnel, «ce jugement» est confié à un traducteur expérimenté. Il existe deux types de révision. La première dite " pragmatique », ne suppose pas de « communication entre le réviseur et l'auteur ou le traducteur » (31). La révision dite " didactique », objet de notre étude, a pour objectif de former ou de perfectionner des traducteurs ou des étudiants (32). Dans ce contexte, la révision de l'enseignant et $\mathrm{du}$ tuteur de stage est une révision didactique qui vise la formation des étudiants/stagiaires.

La révision apporte des corrections au texte traduit : « il s'agit d'une amélioration, et non d'une retraduction ou d'une réecriture » (33). Dans un premier temps, cette correction se base sur la comparaison entre le texte d'arrivée et le texte de départ. A travers cette révision bilingue (34) axée sur l'observation comparative, le sens et la reformulation sont vérifiés (35). A cela s'ajoute la vérification de l'équivalence (36). 
La révision comparative est suivie de la révision unilingue du texte d'arrivée pour le vérifier du point de vue fond et forme (37). Dans ce sens, le réviseur «[pratique] l'empathie (aptitude à se mettre à la place du destinataire), [s'assure] que le texte révisé a atteint la cible, c'est-à-dire qu'il sera lu et provoquera la réaction attendue ». (38). Un des buts de la révision professionnelle est « de rendre acceptable au regard des normes professionnelles les traductions réalisées par d'autres personnes » (39). Dans ce contexte, les notions d'acceptabilité et d'erreur sont liées à la révision. Ce qui peut être accepté par un client, ne l'est pas forcément par un autre.

Les paramètres de la révision professionnelle s'articulent autour de cinq éléments : l'exactitude (fidélité au sens), la correction concernant le code linguistique, la lisibilité liée à la logique, à la clarté et à la concision, la tonalité (registre de langue) et l'adaptation au destinataire notamment culturelle (40).

Certes, les critères de révision sont suivis par les réviseurs mais il arrive que ces derniers soient subjectifs et aient des préférences concernant le style (tournures de phrases ou de verbes) (41) ou les variantes terminologiques (42). La révision, «garante de l'acceptabilité » (43), tient compte de cinq critères qui assurent la qualité :

« transmettre exactement le message de l'original ; 2) observer les normes grammaticales de son temps ; 3 ) être idiomatique ; 4) être dans le même ton que l'original (équivalence stylistique) ; 5) être pleinement intelligible pour le lecteur qui appartient à une autre culture (adaptation culturelle) » (44).

La révision est un élément clé qui s'inscrit dans une optique de qualité. C'est pourquoi il est important d'initier et d'entraîner les étudiants aux critères de la révision : «former les étudiants traducteurs à une bonne pratique de la relecture et de la révision est un minimum. Cela reflète une démarche proactive, qui peut avoir un effet boule de neige : plus on leur apprend à se poser les bonnes questions sur un texte et mieux ils appliqueront cette méthode. » (45).

\subsection{Notions d'erreur et d'acceptabilité}

Les notions d'erreur et d'acceptabilité sont étroitement liées au Cahier des charges. Avant d'entamer la traduction, le traducteur définit sa stratégie générale en fonction des consignes du donneur d'ouvrage. La terminologie et la phraséologie maison ainsi que le guide de style sont pris en compte dans la traduction et dans la révision. Ne pas établir une stratégie de traduction en fonction des objectifs de traduction est l'une des «causes majeures d'erreur en traduction» (46). Certes, la stratégie de traduction est tributaire du destinataire et de la fonction du texte traduit (47). D'où la relativité de la notion d'erreur comme le montre la théorie du skopos (48) dans son lien avec l'acceptabilité. En effet, cette dernière a comme source le cahier des charges : «Ce qui gouverne la notion d'erreur ou de faute en traduction ce ne sont ni l'original ni les normes de la langue ou de la culture d'arrivée à eux seuls, mais 
La qualité de la traduction spécialisée : révision dans les contextes pédagogique et professionnel

aussi la tension qu'exercent simultanément ces derniers sur le texte traduit en fonction des besoins et des valeurs exprimés par le donneur d'ouvrage » (49). Donc, si ce qu'un traducteur peut concevoir comme erreur est ce que le client exige, ce n'est plus considéré comme étant une erreur ; " autrement dit, il ne devrait pas y avoir de faute là où il y a contrainte (par exemple, lorsqu'une terminologie maison est imposée par le client) » (50).

L'erreur en traduction est définie comme étant « une rupture de congruence dans le passage d'un document premier (à traduire, existant, compris, analysé) à un document second (à venir) » (51). Identifier le type d'erreur est d'une importance primordiale car il « permet d'affiner la méthodologie en appliquant une stratégie de résolution adaptée à un type particulier de problème clairement identifié » (52). Les erreurs sont classées en trois catégories : langue, sens et respect des consignes (53). Autrement dit, est considérée comme une erreur toute transgression de la convention de la langue, du sens du texte original et des exigences du donneur d'ouvrage (cahier des charges).

Comme la notion d'erreur, celle d'acceptabilité est relative: «Dans les milieux professionnels, et tout particulièrement dans l'industrie, l'acceptabilité est une notion clé qui détermine la réception d'un produit ou d'un service et qui conditionne son succès commercial. Tout est fait pour réduire son caractère relatif et subjectif, à commencer par la définition et l'établissement de normes. ». (54).

La prise en compte du contexte de l'erreur s'avère être un élément important dans l'étude de l'erreur et de l'acceptabilité de la traduction dans le contexte didactique de la traduction: "l'erreur est un indice précieux permettant à l'enseignant de comprendre le processus d'apprentissage de l'apprenant et de repérer les difficultés de ce dernier » (55). Appuyons nos propos avec les cas pratiques qui suivent.

\section{Cas pratiques :}

Les exemples sélectionnés ci-dessous des cours et des rapports sont analysés en fonction des trois éléments qui conditionnent la qualité de la traduction mentionnés par Brunette dont le destinataire de la traduction, le rôle du texte à traduire et le cahier des charges (56).

\subsection{Public Visé}

\section{Exemple 1 : spécialiste, profane ou néophyte ?}

Il existe pour le terme « système embarqué » une pluralité d'équivalents en arabe. Il s'agit du rapport rédigé en 2008 par le sénateur Claude Saunier, intitulé «L'industrie de la microélectronique : reprendre l'offensive » (57). La consigne donnée pendant le cours concerne la traduction de la synthèse du rapport (exercice de synthèse croisée) destinée aux étudiants de prépa de la faculté d'Ingénierie d'une université publique en Égypte. La traduction paraitrait dans la revue destinée aux 
étudiants pour les informer du contenu du rapport. Il s'agit, donc, d'une vulgarisation du français vers l'arabe.

Quatre équivalents du terme «système embarqué» ont été proposés par les étudiants :

الأنظمة المدمجة

الأنظمة المدموجة

الأنظمة المتكاملة

الأنظمة النقالة

Chaque étudiant est invité à argumenter son choix et à en expliquer les motifs (58).

La fréquence du terme dans les contenus arabes en ligne en est un. Les sources consultées par les étudiants sont les forums et les blogs. Quoiqu'elles ne soient pas crédibles, ces sources ont été le seul recours, vu l'absence de documentation concernant la nanotechnologie en langue arabe. La révision, guidée par l'approche méthodologique a repéré un élément manquant : la consultation d'un spécialiste du domaine de l'électronique. Une semaine plus tard, les étudiants arrivent avec un terme validé par un enseignant à la faculté d'Ingénierie de l'Université française d'Egypte : الأنظمة المدمجة

Le cours de révision de traduction se pratique en groupes. Son objectif est double : appliquer la méthodologie de la traduction (ici, l'accent est mis sur la recherche documentaire du domaine spécialisé et le recours au spécialiste) et initier les étudiants à la révision de la traduction. La révision du texte traduit est effectuée soit par chaque étudiant soit par groupe d'étudiants puisque certaines traductions sont effectuées par groupes (de 2 à 3 étudiants) : « les exercices collectifs permettent aux étudiants d'aborder la révision dans un contexte convivial et stimulant » (59). Le travail en groupes permet de se répartir les tâches de l'étape de pré-traduction, de la traduction et de révision. Cette dernière est effectuée par chaque membre de l'équipe en échangeant les traductions et après que l'auto-révision soit pratiquée : «en enseignement de la traduction, la révision est une activité d'apprentissage qui permet aux étudiants de relever les erreurs ou les maladresses d'une traduction, d'en déterminer la nature et la cause et de faire ressortir également les qualités du texte traduit. » (60).

L'exercice de vulgarisation est pratiqué dans les stages notamment au sein de l'Organisation mondiale de la santé (OMS) et à l'hôpital Egypt Air dans le cadre de la coopération signée en 2012 avec l'hôpital Européen Georges-Pompidou en France. Dans ce contexte, les textes vulgarisés visent à sensibiliser les gens vis-à-vis des habitudes d'hygiène et de santé. Les termes médicaux sont remplacés par des vocables faciles à comprendre comme le remplacement du terme «Polydipsie » par le vocable «soif excessive» plus facile à comprendre par le destinataire. La 
La qualité de la traduction spécialisée : révision dans les contextes pédagogique et professionnel

traduction d'une brochure du français vers l'arabe concernant le dépistage du cancer du sein a amené la stagiaire à utiliser des phrases simples pour vulgariser le message médical tout en le présentant sous forme de questions-réponses. Pour les textes à vulgariser, la consigne est importante. Elle doit être précise et bien définie en déterminant le destinataire, la fonction du texte traduit et le nombre de mots.

\section{Exemple 2 Quelle zone géographique?}

Certes, l'usage est un «concept mouvant : il varie selon l'époque et le lieu » (61). Voici un exemple. Dans un texte publié en 2015 intitulé «Plan national de dématérialisation des marchés publics » (62), l'équivalent arabe du terme «marché public » représente un intérêt particulier et interpelle aussi bien l'étudiant que l'enseignant. Trois variantes sont proposées par les étudiants :

الصفقات العدومية

الأسواق العامة

المشتريات الحكومية

Lors de l'argumentation de leurs choix, les étudiants ont mentionné que le terme « ا المشتريات الحكومية est utilisé en Égypte lorsque le gouvernement (dans le domaine des marchés publics) publie un appel d'offres. Il choisit l'offre et le produit qu'il veut et la technologie. Les étudiants ont trouvé le terme sur le site égyptien du marché public (https://etenders.gov.eg/) qui correspond au sujet du texte. Il existe en deux versions arabe et anglaise. Ils ont trouvé également le terme " الأسواق العامة couvrant le même concept du terme 《 المشتريات الحكومية.

Les étudiants qui ont choisi الصفقات العمومية ont avancé l'argument de l'usage du terme sur le site du Maghreb (https://www.marchespublics.gov.ma/pmmp/?lang=ar $\mathrm{xx}$ ) appuyé par une version bilingue français-arabe, ce qui représente, à leurs yeux, une source crédible pour leur recherche terminologique.

Si on analyse le choix des termes par rapport aux notions d'erreur et d'acceptabilité, il s'avère que le terme 《الصفقات العمومية considérée comme une erreur par l'enseignant égyptien, dans le cadre du cours se déroulant au sein de la faculté des Langues Appliquées, en Egypte, se trouverait accepté dans le cadre d'un cours se déroulant au sein d'une faculté de Langues au Maghreb. Dans ce contexte, la notion d'erreur et celle d'acceptabilité sont traitées en fonction du destinataire se situant dans une zone géographique donnée. En Égypte, les deux termes 《 الأسواق العامة et et ال l'est au Maghreb. Le choix du terme est, donc, tributaire de la région du destinataire. L'usage régional l'emporte et il convient de définir dès le début le destinataire de la traduction. Il faudra attirer l'attention pendant le cours sur le destinataire du texte traduit pour assurer le choix de la variante terminologique. 
Les étudiants qui effectuent leurs stages à l'étranger sont souvent confrontés à la notion de l'usage régional. En voici un exemple tiré du rapport de stage effectué au sein du bureau de traduction Ben Boucetta à Paris dont le directeur est algérien. Il s'agit d'un contrat de mariage traduit du français vers l'arabe. Le dentinaire est le consulat du Royaume du Maroc. Le terme «Carte d'identité» traduit par la stagiaire 《البطاقة الثخصية a été considéré comme une erreur par le tuteur qui l'a

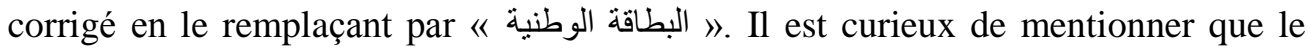
terme « البطاقة الثخصية, d'usage en Egypte, a été remplacé en janvier 2007 par le terme 《ب بطاقة الرقم القومي (traduction littérale : numéro national) qui correspond aux 14 chiffres inscrit sur la carte d'identité digitale. A travers ces chiffres, il est possible d'accéder aux données du citoyen. Il en est de même pour le terme « Euro », traduit

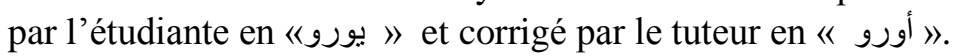

Ce que nous remarquons dans les cours de $4^{\mathrm{e}}$ année, c'est que les étudiants qui ont fait leur stage participent au cours en «[faisant] appel à l'ensemble de [leurs] connaissances, de [leurs] compétences et de [leur] expérience. » (63). A travers les exemples que nous venons d'analyser, il advient que les étudiants ont mis en application lors de leur stage les connaissances acquises en matière de méthodologie de la traduction, de la recherche documentaire, de terminologie et d'informatique.

\subsection{Exigences du donneur d'ouvrage :}

\subsubsection{Glossaire terminologique}

\section{Exemple 1 : Organisations internationales}

Les stagiaires effectuent au Bureau régional de la Méditerranée à l'OMS des traductions de l'anglais vers l'arabe et de l'anglais vers le français. Le passage de l'anglais vers l'arabe et de l'anglais vers le français implique dans certaines situations le recours aux trois langues (anglais, français et arabe). Trilingues, les étudiants s'intègrent sans difficultés dans le service de traduction de l'Organisation. Les textes portent sur des sujets relatifs à la santé. Il s'agit d'articles web, de lettres officielles, de rapports, d'annexes et de textes alternatifs (64). Les stagiaires effectuent également des exercices de révision de traduction.

Les rapports de stages abondent en exemples concernant la nécessité de se conformer au glossaire terminologique de l'Organisation. Prenons l'exemple du terme «food safety» (sécurité sanitaire des aliments) traduit de l'anglais vers l'arabe par la stagiaire 《 الأمان الغذائي (». Le tuteur de stage l'a considéré comme une erreur et l'a corrigée en la remplaçant par le terme «مأمونية الغذاء. Lors de la soutenance du rapport de stage, l'étudiante décrit la démarche suivie pour choisir le terme 《الأمان الغذائي 》. D’abord, elle a cherché la définition du terme anglais «food safety » en anglais et du terme français (sécurité sanitaire des aliments). Elle a effectué une recherche ciblée sur les deux termes en se basant sur les acquis du cours de terminologie. 
La qualité de la traduction spécialisée : révision dans les contextes pédagogique et professionnel

Examinons le terme « sécurité sanitaire des aliments » dans le glossaire de l'Institut de l'information scientifique et technique du CNRS :

« La sécurité sanitaire des aliments a pour objet l'hygiène et l'innocuité des aliments par la règlementation et le contrôle des filières agroalimentaires (contaminants toxiques, agents pathogènes...). On la dénomme également "innocuité des aliments" (Commission générale de terminologie et de néologie) ou "salubrité alimentaire" (FAO). Elle est parfois considérée comme une composante de la sécurité alimentaire au sens large. »(65).

En nous basant sur la définition ci-dessous, cherchons la définition du terme «Sécurité alimentaire » dans la même source pour saisir la différence entre les deux termes :

« disponibilité de produits alimentaires en quantité suffisante pour assurer l'approvisionnement d'une population et subvenir à ses besoins nutritionnels. »(66).

Donc, la distinction des deux termes « sécurité sanitaire des aliments » (en anglais food security) et « sécurité des aliments » (en anglais food safety) éclaire sur le choix de l'équivalent arabe après la consultation de ressources en langue arabe portant sur la sécurité alimentaire des aliments. La stagiaire trouve l'équivalent de 《Sécurité alimentaire» الأمن الغذائي (67) Pour 《 sécurité sanitaire des aliments 》, elle trouve trois termes courants :

(d'usage au sein de l'OMS et dans la presse égyptienne) (68).

الأمان الغذائي/ أمان الغذاء (d’usage dans la presse égyptienne) (69)

المأمونية الغذائية (d'usage au sein de l'OMS)

La définition du terme en arabe « أمان الغذاء est accompagnée du terme anglais (Food Safety) dans le livre intitulé كتاب ادارة الجودة والأمان الغذائس (Livre de la gestion de la qualité et de la sécurité alimentaire) :

$$
\text { 》 هى جميع الإجر اءات اللازمة لإنتاج غذاء صحى غير ضار بصحة الإنسان 《) (70). }
$$

(Traduction de la définition : Toutes les procédures nécessaire à la production des aliments saints non nuisibles à la santé de l'homme)

Observons plus particulièrement l'usage des termes « مأمونية 》 dans le cadre de la célébration de la journée mondiale de la Santé le 7 avril 2015 dans la presse égyptienne. Les journaux égyptiens, Al Doustour et Al Masry Al Yom, par exemple, traitent dans leurs titres de la célébration de la journée en utilisant le terme "الغذاء أمان qui est d'usage en Egypte. Mais, lorsqu'il s'agit des recommandations de

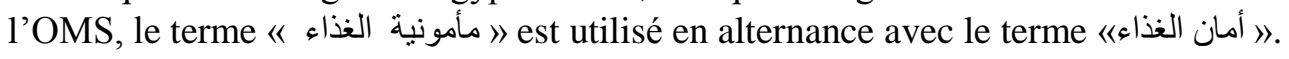


Une discussion avec le tuteur syrien (71) nous renseigne sur les motifs de sa correction. Selon lui, le terme 《 مأمونية الغذاء est le terme consacré puisqu'il couvre exhaustivement toutes les phases concernant l'hygiène et l'innocuité des aliments de la culture à la consommation.

Certes, les étudiants sont sensibilisés, dans les cours de traduction vis-à-vis de l'importance de la consultation de la base de données terminologiques de l'ONU UNTERM (http://untermportal.un.org).

\section{Exemple 2 : Société}

Avançons, dans ce sens, l'exemple des stages effectués à la société Arabize (72), spécialiste dans la gestion du contenu arabe (http://www.arabize.com). Ses activités sont la traduction et la localisation. Ses clients sont internationaux comme Microsoft et Oracle. Les exemples avancés dans ce sens concernent les termes exigés par le commanditaire. Prenons l'exemple d'un projet dans lequel le terme 《) est exigé comme équivalent à « create » (créer) ; alors que dans un autre projet, l'emploi du terme 《 تكوين " est exigé pour le même terme anglais «create». Si pour ce deuxième projet, les stagiaires utilisent le terme 《إنشاء \) (exigé par le commanditaire du premier projet) au lieu du terme 《تكوين cela est considéré comme étant une erreur. Il en est de même pour le verbe «Go to » ayant deux équivalents pour deux projets différents, l'un « انتقال et l'autre تنقل \& De plus, si les traducteurs au sein de la société n'utilisent pas la terminologie inscrite dans le cahier des charges, leur évaluation à la fois par le commanditaire et par la société risque d'être négative allant même jusqu'à leur sanction.

\subsubsection{Guide de style}

Les institutions mettent à la disposition des stagiaires leurs guides de style (73). Ce guide concerne le style adopté par l'institution dans sa rédaction et sa traduction. Il s'agit des préférences stylistiques et des structures de phrases, entre autres. Nous remarquons que le style pompeux en arabe est maintenu dans la traduction française dans les ministères égyptiens, par exemple. Les phrases restent longues et complexes.

Prenons l'exemple de la société Arabize. Les stages effectués au sein de cette société offrent des exemples concernant le style dans la traduction du domaine technique de l'anglais vers l'arabe. Dans le tableau 1 ci-dessous, les syntagmes soulignés traduits par les stagiaires ont été considérés comme une erreur et ont été corrigés par le tuteur. 
La qualité de la traduction spécialisée : révision dans les contextes pédagogique et professionnel

Tableau 1 - Guide de style

\begin{tabular}{|c|c|c|c|}
\hline $\begin{array}{l}\text { Langue } \\
\text { source }\end{array}$ & $\begin{array}{c}\text { Traduction du/de la } \\
\text { stagiaire }\end{array}$ & $\begin{array}{l}\text { Correction } \\
\text { du tuteur }\end{array}$ & Commentaire \\
\hline $\begin{array}{l}\text { Not all } \\
\text { file types } \\
\text { can be } \\
\text { edited }\end{array}$ & لايمكن تحرير بعض الملفات & قا بتعضر تحرير & $\begin{array}{l}\text { Usage du verbe "يتعذر" "يمكن" à la } \\
\text { place du verbe la } \\
\text { notion du conditionnel "قد " } \\
\text { pour désigner un acte } \\
\text { éventuel. }\end{array}$ \\
\hline $\begin{array}{l}\text { Display } \\
\text { other } \\
\text { options }\end{array}$ & اعرض لي خيار ات أخرى & عرض خيارات & $\begin{array}{l}\text { Usage du nom à la place du } \\
\text { verbe à l'impératif. }\end{array}$ \\
\hline $\begin{array}{l}\text { Click } \\
\text { View } \\
\text { Menu }\end{array}$ & انقر على عرض القائمة & عرض القائمة" " النقر " & $\begin{array}{l}\text {-Usage du nom à la place du } \\
\text { verbe à l'impératif. } \\
\text {-Ajout des guillemets pour } \\
\text { l'intitulé de la boite de } \\
\text { dialogue. } \\
\text {-Remplacement de la } \\
\text { préposition «sur » par } \\
\text { «dessus». }\end{array}$ \\
\hline
\end{tabular}

Dans la correction du tuteur, l'impératif en anglais utilisé par la stagiaire 《رض اعرض إن

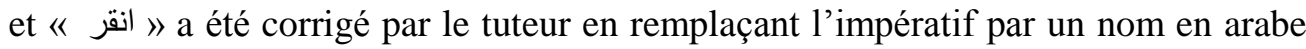
《 " عرض/ Selon le guide de style de l'entreprise, lorsqu'il s'agit de traduire les boites de dialogue vers l'arabe, l'usage du nom est exigé. Autre exigence : les termes doivent être écrits entre guillemets lorsqu'il s'agit d'orienter l'utilisateur vers une option et que ces termes sont en majuscules en langue anglaise comme dans l'exemple de «Click View Menu ». Le tuteur de stage remplace la préposition en arabe (على) 《sur » par la préposition « dessus 》 (فوق) pour le terme النقر فوق car c'est la collocation d'usage au sein de la société.

Les stagiaires attirent l'attention sur l'usage de la phrase nominale en arabe composé du sujet ( المبتدأ privilégié à l'emploi des particules d'objet (إنبر) et du prédicat (الخبر ) (واخواتها ( alors que les étudiants ont l'habitude d'opter pour la phrase verbale en arabe suite aux recommandations des enseignants de langue arabe au sein de la Faculté.

\subsection{Fonction du texte traduit :}

\section{Exemple 1 : Annonce publicitaire}

Avançons dans ce sens l'exemple du stage effectué à Al Ahram Hebdo. Il s'agit de la traduction d'une publicité de l'arabe vers le français. La stagiaire fait un premier jet qu'elle revoie en fonction des commentaires de la tutrice. Le deuxième jet se 
présente comme une version réfléchie répondant à la fonction du texte traduit. Comparons dans le tableau 2 ci-dessous les deux jets.

Tableau 2 - Jets de traduction

\begin{tabular}{|c|c|c|}
\hline Texte source & Premier jet de traduction & Deuxième jet de traduction \\
\hline 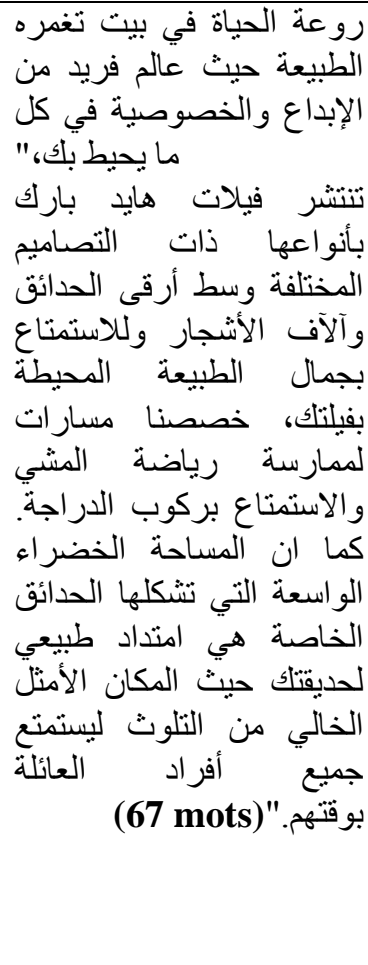 & $\begin{array}{l}\text { La vie en rose dans une } \\
\text { maisen au-sein de la nature, } \\
\text { entourée par un espace de } \\
\text { créativité et d'intimité. Les } \\
\text { différents modèles des } \\
\text { villas Hyde Park sont } \\
\text { réparties sur des parcs les } \\
\text { plus prestigieux entre des } \\
\text { milliers d'arbres. Pour jouir } \\
\text { des merveilleux paysages } \\
\text { naturels enteurant vos } \\
\text { villas, des allées ont été } \\
\text { mises à votre disposition } \\
\text { pour le jogging et les vélos. } \\
\text { Le vaste espace de ces pars } \\
\text { privés est le prolongement } \\
\text { naturel de votre jardin. La } \\
\text { place idéale, loin de la } \\
\text { pollution, où tous les } \\
\text { membres de la familles } \\
\text { passeront de bons moments. } \\
\text { (94 mots) }\end{array}$ & $\begin{array}{l}\text { villas Hyde Park entourées } \\
\text { par un espace de créativité et } \\
\text { d'intimité. Différents } \\
\text { modèles répartis sur des } \\
\text { parcs prestigieux au milieu } \\
\text { des arbres. Merveilleux } \\
\text { paysages, allées de jogging } \\
\text { et de vélo, vaste espace vert, } \\
\text { c'est le prolongement } \\
\text { naturel de votre } \\
\text { jardin...Passez de bons } \\
\text { moments avec toute la } \\
\text { famille (p 23) } \\
\text { (47 mots) }\end{array}$ \\
\hline
\end{tabular}

Ce texte compte dans la version arabe 67 mots. Traduit en français, le premier jet compte 94 mots. Dans son deuxième jet, après les commentaires de la tutrice, le texte est réduit à 47 mots. Les différences entre les deux jets de la stagiaire nous interpellent.

Contrairement au premier jet «collé » au texte source, le deuxième jet tient compte du type de texte et de la fonction du texte traduit. Il s'agit d'une publicité rédigée en arabe. Le texte publicitaire est accompagné du logo de l'entreprise. L'objectif du texte traduit est d'inciter le lecteur à acheter une villa à Hyde Park. La stagiaire a, donc, changé sa stratégie de traduction en retravaillant son $1^{\mathrm{er}}$ jet. Le deuxième jet se focalise sur l'idée de convaincre rapidement et succinctement en adoptant un message concis. Cette concision se manifeste par l'élimination de l'article dans « les différents modèles » et la suppression de certains syntagmes du premier jet (« la vie en rose dans une maison au sein de la nature », «le vaste espace de ces parcs privés »). La reformulation est plus ciblée dans le deuxième jet, ce qui se manifeste par l'usage de l'impératif pour attirer l'attention et convaincre "passez de bons moments ». Dans ce contexte, le deuxième jet garantit l'efficacité du message. 
La qualité de la traduction spécialisée : révision dans les contextes pédagogique et professionnel

La stagiaire a révisé son texte avec soin. Les coquilles, les fautes de grammaire (comme " modèles des villas sont réparties ») et les maladresses de langues sont corrigées (comme dans l'usage impropre du verbe «jouir»). Cette erreur, dûe à «l'insuffisance de maitrise de la langue d'arrivée» (74), risque de compromettre le message publicitaire. Si on consulte le sens du verbe «jouir» dans le cnrtl (http://www.cnrtl.fr/definition/jouir) nous trouvons parmi ses acceptions :

-« Jouir de quelque chose. Éprouver de la joie, du plaisir, un état de bienêtre physique et moral procuré par quelque chose.

-Jouir de quelqu'un. Disposer de quelqu'un afin de combler ses désirs et de satisfaire ses besoins sexuels ».

-Dans le domaine de droit: «jouir d'un droit». Être titulaire d'un droit. Dans le sens d'avoir un avantage. ».

La stagiaire analyse son erreur et la commente en précisant que le verbe «jouir », étant polysémique (dans la langue courante a une connotation sexuelle), son emploi risque de gêner le lecteur. Son usage s'avère être inacceptable en français dans ce contexte publicitaire, comme le lui a bien expliqué sa tutrice. Cet exemple montre l'importance de l'auto-révision et de la révision effectuées en fonction du but du texte à traduire.

\section{Exemple 2 : article argumentatif}

La version française de l'article de l'écrivain Mohamed Salamawy (75) parue dans Al Ahram Hebdo (numéro 723 du 16-22 juillet 2008), intitulé «La déviation religieuse et l'expérience tunisienne» publié sur le lien http://hebdo.ahram.org.eg/Archive/2008/7/16/opin1.htm a été effectuée par notre stagiaire, corrigée par la tutrice et revue par l'auteur lui-même. Examinons le tableau 3 ci-dessous qui contient le sixième paragraphe de l'article et nous interpelle au niveau de la reformulation. 
Tableau 3 - Reformulation

\begin{tabular}{|c|c|c|}
\hline Texte source & Traduction de la stagiaire & \\
\hline 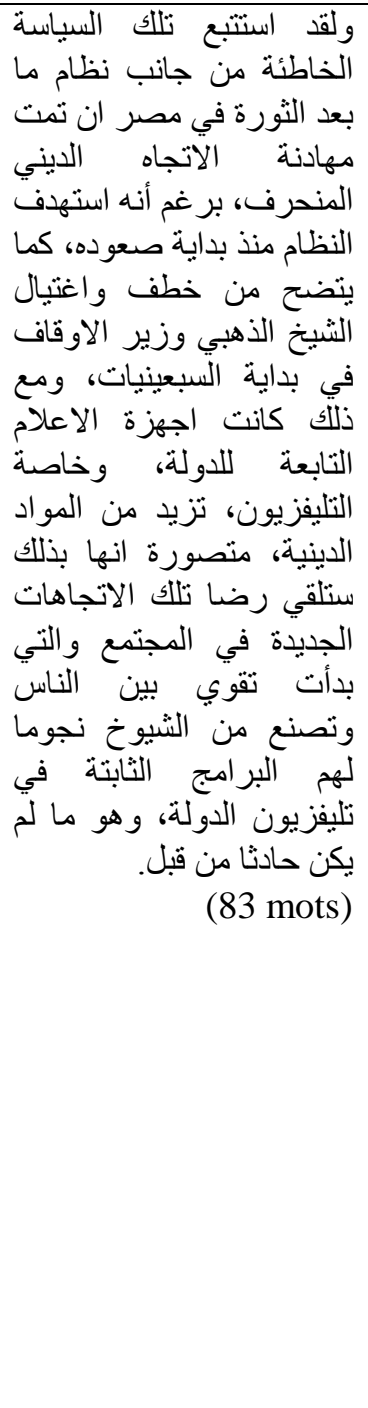 & $\begin{array}{l}\text { «Après cette politique } \\
\text { erronée de la part du } \\
\text { gouvernement égyptien post } \\
\text { révolutionnaire, une } \\
\text { réconciliation a eu lieu avec } \\
\text { la tendance religieuse déviée, } \\
\text { bien qu'elle ait visé le } \\
\underline{\text { système depuis sa montée }} \\
\text { avec l'assassinat et le } \\
\text { kidnapping de Cheikh } \\
\underline{\text { Dahabi, ministre des waqfs }} \\
\text { au début des années 70. } \\
\text { Malgré cela, les moyens } \\
\text { d'information appartenant à } \\
\text { l'Etat, notamment la } \\
\text { télévision, avaient augmenté } \\
\text { le nombre des programmes } \\
\underline{\text { religieux, croyant ainsi qu'ils }} \\
\underline{\text { obtiendraient la bénédiction }} \\
\text { des nouvelles tendances. } \\
\text { Ces dernières commençaient, } \\
\text { en effet, a gagné plus poids } \\
\underline{\text { au sein de la société, et }} \\
\text { faisaient des Cheikhs des } \\
\text { stars des programmes de la } \\
\text { chaine nationale. C'est un } \\
\underline{\text { incident exclusif. " }} \\
\text { (104 mots) }\end{array}$ & $\begin{array}{l}\text { «Cette politique erronée } \\
\text { adoptée par le régime de } \\
\text { l'après-guerre en Egypte, } \\
\text { a été suivie par une autre de } \\
\text { réconciliation avec cette } \\
\text { tendance religieuse déviée, } \\
\text { bien qu'elle ait eu pour } \\
\text { cible le régime depuis son } \\
\text { ascension, comme il est } \\
\text { apparu dans le kidnapping } \\
\text { et l'assassinat de cheikh Al- } \\
\text { Zahabi, le ministre des } \\
\text { Waqfs au début des années } \\
\text { 1970. En dépit de cela, les } \\
\text { organismes médiatiques } \\
\text { étatiques et notamment la } \\
\text { télévision diffusaient } \\
\text { davantage les programmes } \\
\text { religieux, imaginant qu'ils } \\
\text { allaient de cette manière } \\
\text { s'attirer le consentement } \\
\text { des nouvelles tendances } \\
\text { dans la société, qui ont } \\
\text { commencé à gagner du } \\
\text { terrain parmi les masses. } \\
\text { Ils ont alors fait de leurs } \\
\text { cheikhs des stars dans les } \\
\text { programmes de la } \\
\text { télévision de l'Etat. Chose } \\
\text { qui n'avait pas eu lieu } \\
\text { auparavant". } \\
\text { (122 mots) }\end{array}$ \\
\hline
\end{tabular}

Le texte traite de la tendance religieuse extrémiste en Egypte en l'opposant à la Tunisie. Ce texte est argumentatif et vise, d'une part, à convaincre le lecteur de la nécessité de combattre le courant religieux extrémiste et à suivre l'exemple tunisien en matière de religion, d'autre part. La traduction de la stagiaire représente un intérêt particulier dans la mesure où la reformulation de l'idée dans certains paragraphes du texte nécessite une analyse du point de vue de l'erreur et de l'acceptabilité

L'auto-évaluation de la stagiaire, suite à la lecture de la correction de la tutrice, lui permet de prendre connaissance de ses erreurs. Elle commente en précisant que 
La qualité de la traduction spécialisée : révision dans les contextes pédagogique et professionnel

l'auteur a considéré la réconciliation comme une politique alors que dans la traduction de la stagiaire, la réconciliation a été présentée comme un fait nouveau et non comme une seconde politique tel que le texte de départ l'a présentée : « après cette politique erronée de la part du gouvernement égyptien post révolutionnaire, une réconciliation a eu lieu avec la tendance religieuse déviée ». Ici, il s'agit d'une erreur dûe à « une reformulation insuffisamment attentive du texte d'arrivée » (76). La correction de la tutrice transmet le sens : «Cette politique erronée adoptée par le régime de l'après-guerre en Egypte, a été suivie par une autre de réconciliation avec cette tendance religieuse déviée ». Notons dans ce sens que la tutrice oriente la stagiaire en fonction des conventions de rédaction journalistique au sein d'Al Ahram Hebdo. En plus, l'espace accordé au texte français conditionne la reformulation qui doit être concise et ciblée. La plupart du temps, la traduction des articles consiste en une synthèse déterminée par le nombre de mots du texte à traduire.

Nous remarquons que le paragraphe en arabe est composé d'une seule phrase. Le traduire en français nécessite de respecter les conventions de la langue française. C'est ainsi que la stagiaire la découpe en trois phrases (correspondant chacune a une idée) sans porter atteinte à la logique suivie par l'auteur et ce pour garantir le critère de la cohérence.

L'auto-analyse menée par certains étudiants dans leurs rapports de stage sur les sources et les types des erreurs commis est enrichissante pour les enseignants en vue de mener une réflexion traductologique sur l'erreur et son « rôle productif» si l'on reprend l'idée de Nicolas Froliger :

« Au demeurant, nous entendons bien, dans cette exploration, nous servir de l'erreur: les nôtres ou celles que nous constatons chez des collègues ou des étudiants. Il existe en effet des erreurs intelligentes, c'est-à-dire révélatrices d'un état d'esprit, d'une démarche de pensée qui permet de mieux comprendre ce qu'aurait dû ou pu être une traduction réussie. Loin de les condamner ou de les fustiger, il convient d'en user : elles sont un moyen d'accès à une réflexion argumentée. » (77).

\section{Conclusion}

La problématique de la révision de la traduction spécialisée dans son lien avec les notions de l'erreur et de l'acceptabilité montre à travers cette étude l'importance de l'analyse des rapports de stages des étudiants effectués au terme de la $3^{\mathrm{e}}$ année. Cette analyse est complétée par le contact direct avec les tuteurs de stage dont le retour est bénéfique pour les enseignants. La maîtrise de la méthodologie de la recherche documentaire et celle de l'informatique sont les deux éléments communément appréciés par les tuteurs de stage. Les étudiants de traduction spécialisée suivent des cours axés sur les besoins ciblés en matière d'informatique. La compétence de la recherche documentaire leur permet d'effectuer une recherche pour avoir accès à la terminologie et à la phraséologie de l'institution qui les accueille en stage. C'est ainsi qu'ils peuvent se préparer avant d'entamer leur stage et s'intégrer dans le milieu du travail. Sensibilisés dans les cours de traduction aux 
spécificités de certaines institutions en Égypte, les étudiants ont une idée plus ou moins claire de ce qui les attend dans leur première expérience professionnelle de traduction. La ponctualité, le sens de l'organisation, la créativité, le regard critique et le respect des délais comptent parmi les éléments positifs du retour des tuteurs de stage.

Lors du stage, les étudiants expérimentent la méthodologie de la traduction et de celle de la recherche documentaire. Ils se rendent compte de leur efficacité. Ils comprennent que toutes les matières sont liées : terminologie, traduction, économie, droit et informatique. Ils s'aperçoivent qu'ils gagent en confiance et avouent avoir aimé le métier de traducteur dont la perception n'était pas claire avant le stage. Nous remarquons une sorte de regain d'intérêt pour la traduction en tant que métier. La première expérience professionnelle des étudiants se manifeste par une prise de conscience des acquis méthodologiques à l'université et des métiers de la traduction dans le monde du travail. Ils se rendent de plus en plus compte de l'importance du métier de traducteur et de réviseur.

Confrontées, la révision de l'enseignant et celle du tuteur de stage suivent les mêmes critères malgré la différence de contexte. Les cours participent à une certaine simulation de ce monde réel à travers les projets pédagogiques et les exemples issus des rapports de stage analysés (comme pour les consignes des exercices de vulgarisation). L'expérience des étudiants permet aussi de varier les types et les sources des textes traités dans le cadre des cours.

Avec l'analyse de la révision des tuteurs, il y a un changement de « jugement » de la part des enseignants. La culture académique se trouve enrichie par la culture de l'entreprise. Autrement dit, le recours au glossaire terminologique et au guide de style permet une ouverture aux moyens diversifiés de reformulation d'idées. La révision de la traduction dans le milieu universitaire tient compte des modalités d'évaluation professionnelle pratiquée dans les institutions ayant accueilli les étudiants de Langues Appliquées.

Il serait intéressant de prévoir une coopération entre les mondes académique et professionnel au niveau de la révision dans un objectif de qualité. La mise en place de projets à visée pédagogique avec les institutions qui accueillent nos étudiants en stage permettrait d'enrichir la visée pédagogique. Il serait possible d'envisager un projet regroupant les étudiants, les enseignants et les tuteurs de stage. Les étudiants traduisent, les enseignants révisent et les tuteurs fournissent un retour vis-à-vis de cette révision en fonction des critères de qualité. Il serait intéressant d'examiner la différence entre la révision de l'enseignant de celle du tuteur. Cet exercice permettrait de redéfinir les critères et les modes d'évaluation pédagogiques à la lumière des critères professionnels. Dans ce scénario, la prise en compte de la culture d'entreprise et du cahier des charges sera de plus en plus présente dans 
La qualité de la traduction spécialisée : révision dans les contextes pédagogique et professionnel

l'esprit de l'enseignant. Bref, cette expérience professionnelle permet une ouverture pour les mémoires terminologiques notamment en matière de variantes terminologiques liées au monde professionnel.

\section{Bibliographie :}

Allignol, Claire. 2007. " L'évaluation dans la formation des traducteurs spécialisés. " In Lavault-Olléon, Elisabeth (ed.) Traduction spécialisée: pratiques, théories, formations, Berne : Peter Lang, p. 237-265.

Ammour, Elizabeth. 2002. «La Révision comme outil de réflexion en traduction ». In Israël Fortunato (ed.), «Identité, altérité, équivalence ? La traduction comme relation», Paris : Minard, p. 55-78.

Association Française de Normalisation (AFNOR). 2012. Norme expérimentale XP ISO/TS 11669 X03-016, projet de traduction, lignes directrices générales. France : AFNOR.

Barabé, Donald et Brunette, Louise. 2011. «La normalisation industrielle en traduction : normalisateurs contre formateurs ? »In Gras, Thierry (ed.), Scolia $n^{\circ} 25$, traduction des normes et normes de traduction dans l'espace européen institutions et entreprises, p 45-54.

Barsoum, Yasmine. 2015. «La variation terminologique du terme : obstacle ou atout pour la traduction ? Cas des deux langues française et anglaise » In Callego Daniel, Current Approaches to Business and Institutional Translation, (ed.), Peter Lang, 2015.

Brunette, Louise. 2003. «Révision et mesure de la qualité des traductions ». In Gouadec Daniel (ed.), Traduction, terminologie, rédaction. Actes des universités d'été et d'automne 2002 et du colloque international «spécialités et spécialisations dans la pratique professionnelle et la formation des traducteurs». Paris. La Maison du dictionnaire.

Brunette, Louise. 2007. «Relecture-révision, compétences indispensables du traducteur spécialisé ", In Lavault-Olléon, Élisabeth (ed.), Traduction spécialisée : pratiques, théories, formations, Berne, Peter Lang, p. 225-235.

Collombat, Isabelle. 2009. «La didactique de l'erreur dans l'apprentissage de la traduction ». Jostrans, The Journal of Specialised Translation. 12:37-54. http://www.jostrans.org/issue12/art_collombat.php (Consultée le 25 mars 2017)

Darbelnet, Jean. 1970. «Traduction littérale ou traduction libre ? », Meta, vol. 15, numéro 2, p. $88-94$.

Delisle, Jean, Lee-Jahnke, Hannelore et Cormier, Monique. 1999. Terminologie de la traduction, $\quad$ FIT $\quad$ Monograph Series/Collection https://books.google.com.eg/books?isbn=9027224234 (consultée le 25 mars 2017)

EL Husseini, Dima. 2016. «Le trilinguisme dans le contexte de l'économie émergente : cas de la faculté des Langues Appliquées de l’Université Française d'Egypte. », Congrès 


\section{Dima EL HUSSEINI}

ANLEA-AILEA 2016, Université de La Rochelle. http://portail-video.univ-lr.fr/Letrilinguisme-dans-le-contexte (consultée le 10 mars 2017). Sur itunes https://itunes.apple.com/fr/itunes-u/congres-anlea-ailea-2016/id1129269225?mt=10 (consultée le 10 mars 2017).

EL Qasem, Fayza. 2015. «Compétences rédactionnelles et révision », conférence donnée à L'Ecole supérieure d'interprétation de conférences et de traduction de Saint Petersburg (St Petersburg School of Conference Interpreting and Translation).

Froeliger, Nicolas. 2013. Les noces de l'analogique et du numérique. De la traduction pragmatique, Paris, Les Belles Lettres, coll. «Traductologiques ».

Gauthier, François. 2010. Objectifs clients. Un guide pour traducteurs et autres travailleurs autonomes du domaine langagier. Montréal : Linguatech.

Gile, Daniel. 2005. La traduction, la comprendre, l'apprendre. Paris : Presses Universitaires de France.

Gouadec Daniel. 1989. «Comprendre, évaluer, prévenir : Pratique, enseignement et recherche face à l'erreur et à la faute en traduction. », TTR, vol. 2, numéro 2, p. 35-54.

Gouadec, Daniel. 1990. « Traduction signalétique ». Meta vol. 35, numéro 2, p. 332-341.

Guidère, Mathieu. 2008, La commnunication multilingue. Traduction commerciale et institutionnelle, De Boeck.

Guidère ; Mathieu. 2010. Introduction à la traductologie, De Boeck. $2^{\mathrm{e}}$ édition.

Horguelin, Paul A. et Pharand, Michelle. 2009. Pratique de la révision, Linguatech, $4^{\mathrm{e}}$ édition.

Larose, Robert. 1998, «Méthodologie de l'évaluation des traductions », Meta, vol. 43, numéro 2, p. 163-190. http://www.erudit.org/fr/revues/meta/1998-v43-n2meta171/003410ar/ (consultée le 10 mars 2017).

Lavault-Olléon, Élisabeth. 2006. «Le skopos comme stratégie de déblocage : dialecte et scotticité dans Sunset Song de Lewis Grassic Gibbon », Meta, vol. 51, numéro 3, p. 504-523. http://id.erudit.org/iderudit/013555ar (consultée le 15 février 2017).

Lavault-Olléon, Élisabeth et Allignol, Claire. 2014. « La notion d'acceptabilité en traduction professionnelle : où placer le curseur ? », ILCEA, numéro 19. http://ilcea.revues.org/2455 (consultée le 22 avril 2017).

Lederer, Marianne. 1994. La traduction aujourd'hui. Le modèle interprétatif, Hachette.

Minacori, Patricia. 2005. «Positionnement épistémologique de la traduction scientifique et technique, notion d'objectivité et implications pédagogiques.» Meta, numéro 504. 
La qualité de la traduction spécialisée : révision dans les contextes pédagogique et professionnel

http://www.erudit.org/fr/revues/meta/2005-v50-n4-meta1024/019871ar/resume/ (consultée le 29 avril 2017).

Porcher Louis. 1977. « Note sur l'évaluation ». In Dabène Michel et Porcher, Louis (ed.), Enseignement du français et formation continue des adultes, Langue française, numéro 36, p. 110-115. http://www.persee.fr/doc/lfr_0023-8368_1977_num_36_1_4847 (consultée le 21 avril 2017).

Salamawy ̈̈Mohamed. 2008. «La déviation religieuse et l'expérience tunisienne ». Al Ahram Hebdo (numéro 723, 16-22 juillet). http://hebdo.ahram.org.eg/Archive/2008/7/16/opin1.htm (Consultée le 21 avril 2017).

Saunier, Claude. 2008. «L'industrie de la microélectronique : reprendre l'offensive». https://www.senat.fr/rap/r07-417/r07-417-syn.pdf

(Consultée le 20 mars 2017)

Toury, Gidéon. 1995. Descriptive Translation Studies and Beyond, Amsterdam \& Philadelphia, John Benjamins Publishing Company.

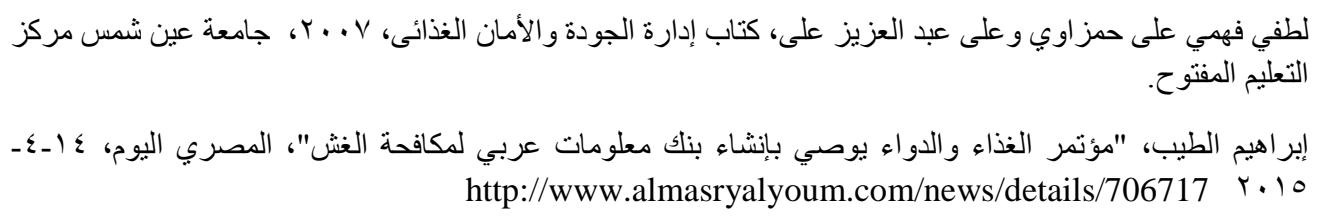

(Consultée le 21 mars 2017)

\section{Notes}

1. De l'anglais vers le français uniquement.

2. La Faculté célèbre la Journée du traducteur depuis 2013.

3. Association Française de Normalisation (AFNOR), Norme expérimentale XP ISO/TS

11669 X03-016, projet de traduction, lignes directrices générales. 2012, France, AFNOR, p. 13.

4. Ibid., p. 19.

5. Lavault-Olléon, Élisabeth. «Le skopos comme stratégie de déblocage : dialecte et scotticité dans Sunset Song de Lewis Grassic Gibbon», Meta, vol. 51, numéro 3, 2006, p. 6.

6. Guidère; Mathieu. Introduction à la traductologie, De Boeck, 2010, $2^{\mathrm{e}}$ édition, p. 101-102.

7. Ibid., p.102.

8. Ibid., p. 102.

9. Lavault-Olléon, Élisabeth, « Le skopos comme stratégie de déblocage : dialecte et scotticité dans Sunset Song de Lewis Grassic Gibbon », Meta, vol. 51, numéro 3, 2006, p. 511.

10. Guidère, Op.cit., 2010, p. 102.

11. Lavault-Olléon, Op.cit., 2006, p. 511. 
12. Horguelin, Paul A. et Pharand, Michelle, Pratique de la révision, Linguatech, $4^{\mathrm{e}}$ édition, 2009 , p. 9.

13. Ibid., p.25 et Brunette, Louise, « Révision et mesure de la qualité des traductions ». In Gouadec Daniel (ed.), Traduction, terminologie, rédaction. Actes des universités d'été et d'automne 2002 et du colloque international «spécialités et spécialisations dans la pratique professionnelle et la formation des traducteurs», Paris. La Maison du dictionnaire, 2003.

14. Horguelin, Paul et Pharand, Michelle, Op.cit., 2009, p.25.

15. Larose, Robert, « Méthodologie de l'évaluation des traductions. », Meta, vol. 43, numéro 2, 1998, p. 6.

16. Ibid., p. 15.

17. Lavault-Olléon, Élisabeth et Allignol, Claire, « La notion d'acceptabilité en traduction professionnelle : où placer le curseur? », ILCEA, numéro 19, 2014, p. 3.

18. Barabé, Donald et Brunette, Louise, « La normalisation industrielle en traduction : normalisateurs contre formateurs ? »In Gras, Thierry (ed.), Scolia ${ }^{\circ} 25$, traduction des normes et normes de traduction dans l'espace européen institutions et entreprises, 2011 , p. 30.

19. Il s'agit de projets de traduction en collaboration avec le département Affaires et Commerce international au sein de la Faculté. Voir « le trilinguisme dans le contexte de l'économie émergente : cas de la faculté des Langues Appliquées de l'Université Française d'Egypte. » http://portail-video.univ-lr.fr/Le-trilinguisme-dans-le-contexte

20. Lederer, Marianne, La traduction aujourd'hui. Le modèle interprétatif, Hachette, 1994, p. 213.

21. Guidère, Mathieu, La commnunication multilingue. Traduction commerciale et institutionnelle, De Boeck, 2008, p.117.

22. EL Qasem, Fayza, «Compétences rédactionnelles et révision », conférence donnée à L'Ecole supérieure d'interprétation de conférences et de traduction de Saint Petersburg (St Petersburg School of Conference Interpreting and Translation), 2015.

23. Collombat, Isabelle, «La didactique de l'erreur dans l'apprentissage de la traduction ». Jostrans, The Journal of Specialised Translation, numéro 12, 2009, p. 40 .

24. Association Française de Normalisation (AFNOR), Op.Cit., 2012, p. 27.

25. Ibid., p. 27.

26. Ibid., 2012, 27.

27. Horguelin Paul et Pharand, Michelle, Op.Cit., 2009, p. 4.

28. Ammour, Elizabeth, «La Révision comme outil de réflexion en traduction ». In Israël Fortunato (ed.), «Identité, altérité, équivalence ? La traduction comme relation», Paris : Minard, 2002, p. 56.

29. Porcher, Louis, « Note sur l'évaluation ». In Dabène Michel et Porcher, Louis (ed.), Enseignement $\mathrm{du}$ français et formation continue des adultes, Langue française, numéro 36, 1977, p. 111.

30. Horguelin, Paul et Pharand, Michelle, Op.Cit., 2009, p. 8.

31. Ibid., p. 3.

32. Ibid., 2009, p. 4.

33. Ibid., 2009, p.1.

34. Ibid, 2009, p.1. 
La qualité de la traduction spécialisée : révision dans les contextes pédagogique et professionnel

35. Delisle, Jean, Lee-Jahnke, Hannelore et Cormier, Monique, Terminologie de la traduction, FIT Monograph Series/Collection, 1999, p. 71.

36. Horguelin Paul et Pharand Michelle, Op.Cit., 2009, p. 3.

37. Ibid., p.1.

38. Association Française de Normalisation (AFNOR), Op.Cit., 2012, p10.

39. Delisle, Lee-Jahnke et Cormier, Op.Cit., 1999, p. 71.

40. Horguelin Paul et Pharand, Michelle, Op.Cit. ; 2009, p. 26 et Association Française de Normalisation (AFNOR), Op.Cit., 2012, p. 24-25.

41. Contact téléphonique avec Dr Yasser Chaddad, chef de la section de traduction arabe au Comité international de la Croix rouge (CICR) à Genève (juin 2015).

42. Voir Yasmine Barsoum, «La variation terminologique du terme : obstacle ou atout pour la traduction? Cas des deux langues française et anglaise », In Current Approaches to Business and Institutional Translation, Daniel Callego (ed.), Peter Lang, 2015.

43. Lavault-Olléon et Allignol, Op.Cit., 2014, p 11.

44. Darbelnet, Jean, « Traduction littérale ou traduction libre ? », Meta, vol. 15, numéro 2, 1970 , p. 89.

45. Minacori, Patricia, «Positionnement épistémologique de la traduction scientifique et technique, notion d'objectivité et implications pédagogiques. », Meta, numéro 504, 2005, p. 13.

46. Gouadec, Daniel, « Traduction signalétique ». Meta vol. 35, numéro 2, 1990, p. 333.

47. Collombat, Op.Cit., 2009, p. 48.

48. Ibid., p. 48.

49. Larose, Op.Cit., 1998, p. 11.

50. Ibid., p. 15.

51. Gouadec, Daniel, 1989, «Comprendre, évaluer, prévenir : Pratique, enseignement et recherche face à l'erreur et à la faute en traduction. », TTR, vol. 2, numéro 2, 1989, p. 38.

52. Collombat, Op.Cit., 2009, p. 47.

53. Lavault-Olléon et Allignol, Op.Cit., 2014, p. 8.

54. Ibid., p. 2.

55. Collombat, Op.Cit., 2009, p 47.

56. Brunette, Louise, « Relecture-révision, compétences indispensables du traducteur spécialisé », In Lavault-Olléon, Élisabeth (ed.), Traduction spécialisée : pratiques, théories, formations, Berne, Peter Lange, 2007.

57. https://www.senat.fr/rap/r07-417/r07-417-syn.pdf

58. Les étudiants soutiennent un mémoire terminologique en $4^{\mathrm{e}}$ année. Ils analysent vingt termes du point de vue terminologique et cinq termes qui posent un problème au niveau des équivalences en traduction : pluralité d'équivalences, variantes régionales et absence d'équivalence.

59. Ammour, Op.Cit., 2002, p. 56.

60. Delisle, Lee-Jahnke et Cormier, Op.Cit., 1999, p.71.

61. Horguelin, Paul et Pharand, Michelle, Op.Cit., 2009, p. 10.

62. https://www.economie.gouv.fr/daj/plan-national-dematerialisation-des-marchespublics

63. Gauthier, François, Objectifs clients. Un guide pour traducteurs et autres travailleurs autonomes du domaine langagier. Montréal, Linguatech, 2010, p. 101. 
64. Textes qui décrivent une image ou une vidéo accompagnant l'article. L'accès au texte alternatif se fait lorsque la souris s'arrête sur l'image. Il est destiné aux non-voyants. Les textes alternatifs commencent souvent par «l'image nous montre ». En voici un exemple : http://www.emro.who.int/fr/surveillance-prevision-et-action/surveillanceevents/ebola-preparedness-meeting.html

65. http://duchampalatable.inist.fr/spip.php? $\operatorname{mot} 114$

66. http://duchampalatable.inist.fr/spip.php?mot111

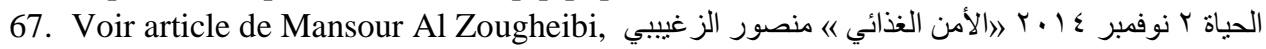

68. http://m.almasryalyoum.com/news/details/706717

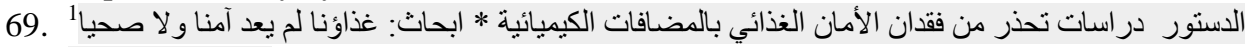

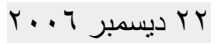

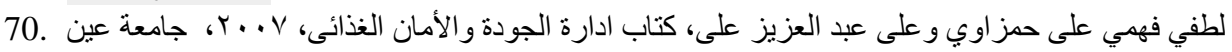

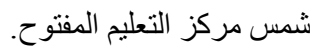

71.

L'OMS utilise

aussi le terme السلامة الغذائية Entretien téléphonique avec le tuteur syrien le 6 mai 2015. Ce terme nous incite à mentionner l'exemple de quelques termes qui sont d'usage uniquement à l'ONU comme ميزنة mayzanna pour « budgétisation » et « sensitivity » (sensibilistation) (au lieu de « awarness »).

72. Arabize a obtenu en 2008 la certification ISO 9001 :2008 et en 2009 la norme européenne de qualité EN $15038: 2006$.

73. ${ }^{1}$ Dont l'agence d'informations MENA, la chaine de télévision ART (Iqraa), la version anglaise du journal Al Masry Al Yom (n'existe plus).

74. Gile, Daniel, La traduction, la comprendre, l'apprendre. Paris : Presses Universitaires de France, 2005, p. 214.

75. version arabe parue dans le journal Al Ahram le 16 juillet 2008 : http://www.ahram.org.eg/Archive/2008/7/16/WRIT2.HTM

76. Gile, Op.Cit., 2005 p 214.

77. Froeliger, Nicolas, Les noces de l'analogique et du numérique. De la traduction pragmatique, Paris, Les Belles Lettres, coll. «Traductologiques », 2013, p. 25-26. 